\title{
Numerical simulation of vertical Bridgman solidification of CdZnTe
}

\author{
Hanen Jamai ${ }^{1}$,*, Bernard Pateyron ${ }^{2}$, Habib Sammouda ${ }^{1}$, and M. El Ganaoui ${ }^{3}$ \\ 1 LabEM (LR11ES34), University of Sousse, ESSTH Sousse, Rue Lamine Abbassi, 4011 Hammam Sousse, Tunisia \\ 2 CNRS-SPCTS/CEC, 12 rue Atlantis, 87068 Limoges, France \\ 3 Université de Lorraine, Lab. Energétique de Longwy - LERMAB/FJV, Longwy, France
}

Received 8 February 2014 / Accepted 17 April 2014

\begin{abstract}
The solidification of the singular CdZnTe in axisymetric Vertical Bridgman (VB) cavity is investigated in the current study. The numerical approach is developed by using COMSOL Multiphysics software, which is based on finite elements method (FEM) with Arbitrary Lagrangian-Euleurian (ALE) formulation allowing treating the moving interface. Numerical aspects of different parameters that affect the solidification interface and present an important factor during the crystal growth's process are presented and analyzed. Especial attention will be paid to investigate the thermophysical properties such as thermal diffusivity, heat capacity. Thermal conductivity and the effect of thermal condition specially the effect of cold temperature $T_{\mathrm{c}}$ which affects significantly the concavity and the convexity of the interface for specific ranges in the solidification of $\mathrm{CdZnTe}$ phenomena, but it is of great importance the crystal growth experts are not it given importance. It has been shown that this properties, the effect of $T_{\mathrm{c}}$, and the variation of interface velocity affect the solid/liquid interface shape and the crystal growth process.
\end{abstract}

Key words: CdZnTe solidification, Axisymetric Bridgman Vertical (BV), ALE formulation.

\section{Introduction}

The vertical Bridgman method for crystal growth from the melt was firstly developed in 1925 . Nowadays, it is the most useful technique for the growth of crystals. This technique is explained in Figure 1. Its perfections to control temperature gradients and to provide provides pure crystals of large sizes by allowing testing the boundary conditions of mass and heat transfer. Hence, such technique is widely utilized in order to produce II-VI compound semiconductor crystals. Their industrial applications are mostly concentrated in detectors and solar cell production fields. Among these semiconductors, the Cadmium zinc telluride $(\mathrm{CdZnTe})$ is considered as one of the most important and promising applied materials for room-temperature $\gamma$ and X-ray spectroscopy. Despite the fact that great efforts have been made to produce high quality of $\mathrm{CdZnTe}$ dominated by the Bridgman method, its production still suffers from small device size and low quality.

The solid-liquid interface shape is specially determined by heat transfer in melt crystal growth process. The search focus in heat transfer during Bridgman growth are presented in references [1-5], these others studied the factors affecting the shape

*e-mail: hanene.jamai@etu.unilim.fr of the liquid-solid interface, such as the such as the thermal conductivities of the crystal, melt, and ampoule, simple furnace characteristics and latent heat generation. In particular, Adornato and Brown [6] examined the effect of furnace profiles and ampoule materials on interface shape and satisfactorily compared their results to experiments of Wang et al. [7]. Jasinski and Witt [8, 9] clarified notable insights on how interface shape may be controlled in Bridgman processes and considered the growth of CdTe as an example system.

In the aim of better understanding the solidification phenomenon and solving the physical problem during the crystal growth, researchers have chosen to test them by implementing a number of different numerical methods that can predict both the shape and position of the phase change front that are compatible with the governing conservation equations in each phase [10]. For instance, one of the most used methods is the front tracking approach which solves weak formulations of these equations by interface tracking by remeshing both states at every temporal step [11]. However, it is known that such method is not appropriate to study solidification process of alloys that undergo a frequent "mushy region" instead of sharp interface. Furthermore, another used method is the enthalpy method which is an approach that fixes the energy equation by using coordinates transformation. On another hand, the 


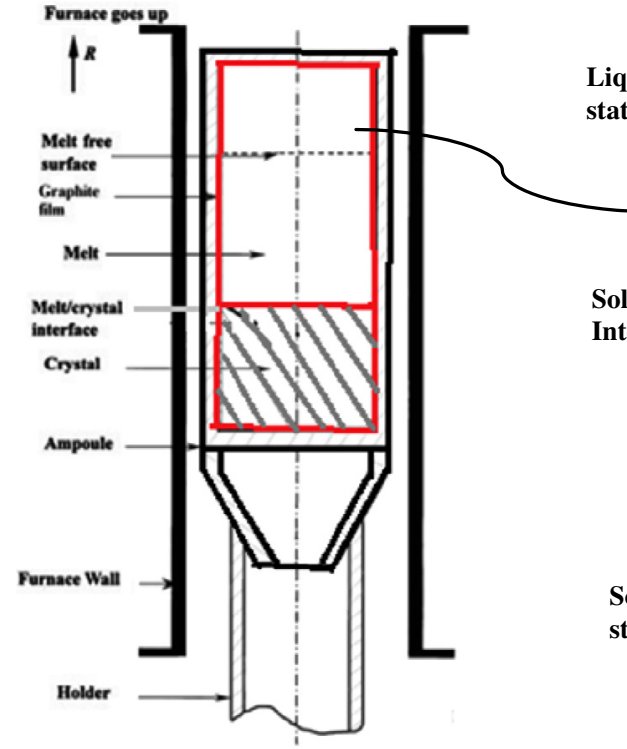

(a)

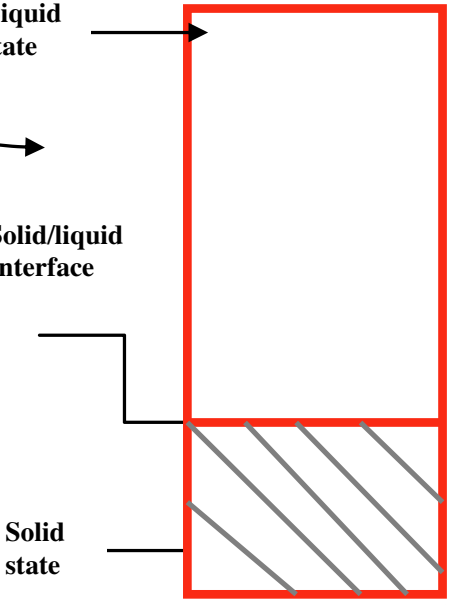

(b)

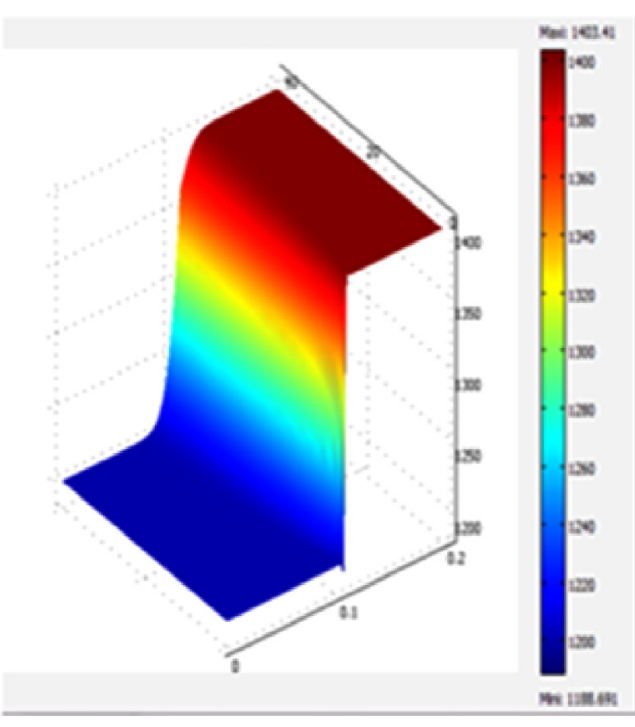

(c)

Figure 1. System. (a) Vertical Bridgman, (b) design domain, and (c) isotherms profile.

Table 1. Physical properties of CdZnTe.

\begin{tabular}{lll}
\hline Property & \multicolumn{1}{c}{ Temperature } & \multicolumn{1}{c}{ Polynomial } \\
\hline Conductivity, $/(\mathrm{K} \mathrm{m})$ & $500 \mathrm{~K}<T<1200 \mathrm{~K}$ & $5.337-2.263 \times 10^{-3} \mathrm{~T}$ \\
& $1200 \mathrm{~K}<T<1364 \mathrm{~K}$ & $-38.31+6.78 \times 10^{-2} \mathrm{~T}-2.811 \times 10^{-5} \mathrm{~T} 2$ \\
Specific heat $C p, \mathrm{~J} /(\mathrm{Kg} \mathrm{K})$ & $T>1364 \mathrm{~K}$ & $9.987-5.676 \times 10^{-3} \mathrm{~T}$ \\
Heat of fusion $\Delta H, \mathrm{~J} / \mathrm{Kg}$ & $298 \mathrm{~K}<T<1600 \mathrm{~K}$ & $177.8+1.663 \times 10^{-1} \mathrm{~T}-6.607 \times 10^{-5} \mathrm{~T} 2+1.703 \times 10^{-8} \mathrm{~T} 3$ \\
Expansion coefficient & 209000 & 209000 \\
Melting point $T_{\mathrm{m}}, \mathrm{K}$ & $T>525 \mathrm{~K}$ & $6.3629 \times 10^{-6}$ \\
Density Kg/m & 1364 & - \\
\hline
\end{tabular}
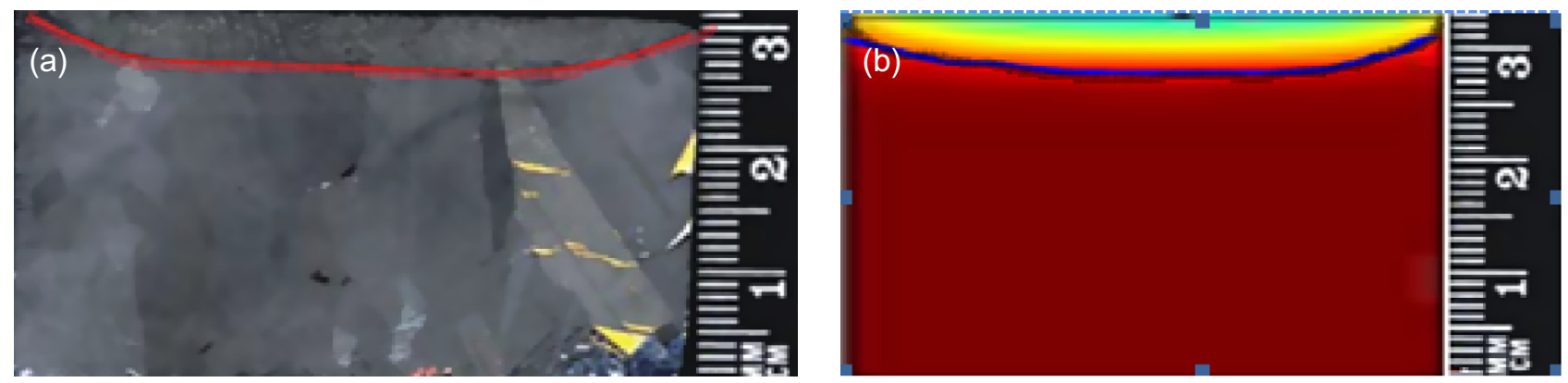

Figure 2. Comparison between experimental data obtained by Sen et al. [18] (a) and our numerical results (b) in terms of the interface growth of CdZnTe.

interface position is recovered a posteriori [11] and an extension of such a front fixing approach has been proposed in literature in order to introduce and predict the convection phenomenon within the two mushy region phases [12-14]. El Ganaoui et al. [15-17] improved this method by implementing a timedependent enthalpy-porosity formulation for directional solidification, in which the energy equation is introduced by using the enthalpy variable computing implicitly the thermal Stefan condition at the interface with an implicit procedure.

The current work uses COMSOL Multi-physics software, to simulate solidification in Bridgman Vertical (BV) configuration during $\mathrm{CdZnTe}$, which is an II-VI compound crystal growth. To the best knowledge of the authors, it seems that thermophysical properties such as thermal diffusivity, heat capacity, 
H. Jamai et al.: Int. J. Simul. Multisci. Des. Optim. 2014, 5, A23

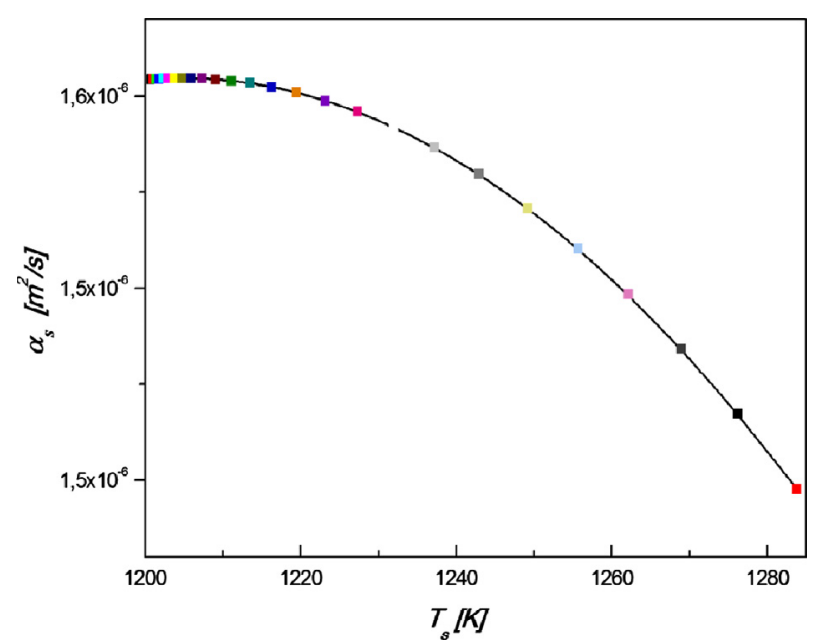

(a)

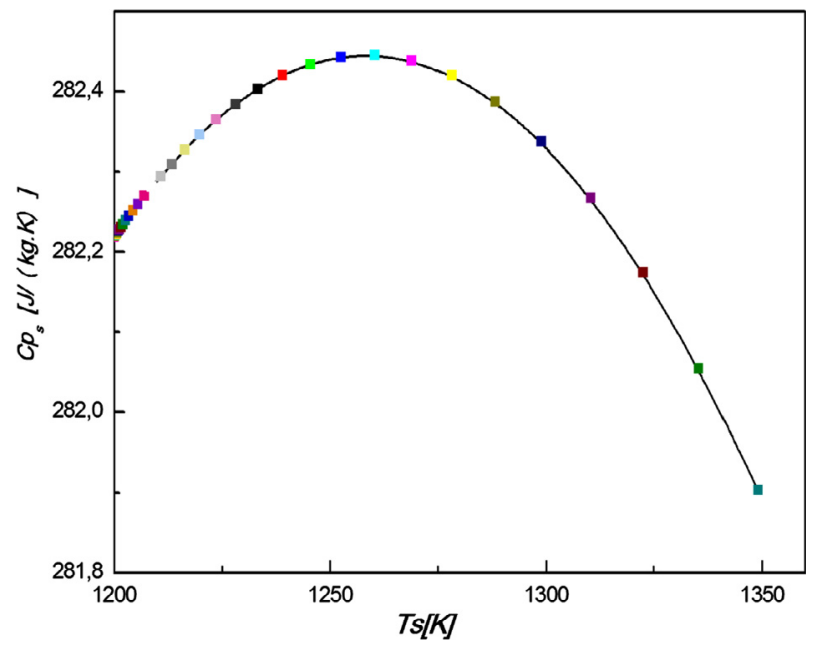

(c)

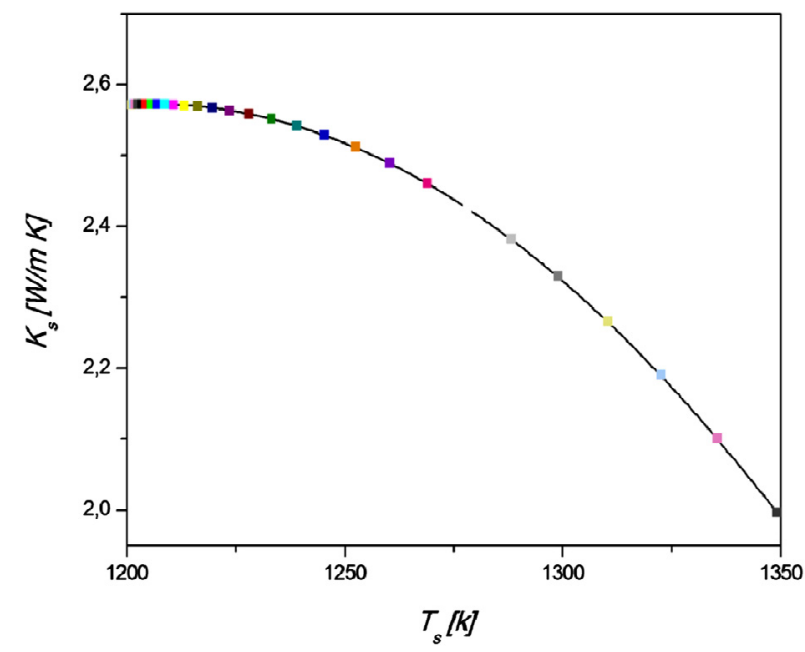

(e)

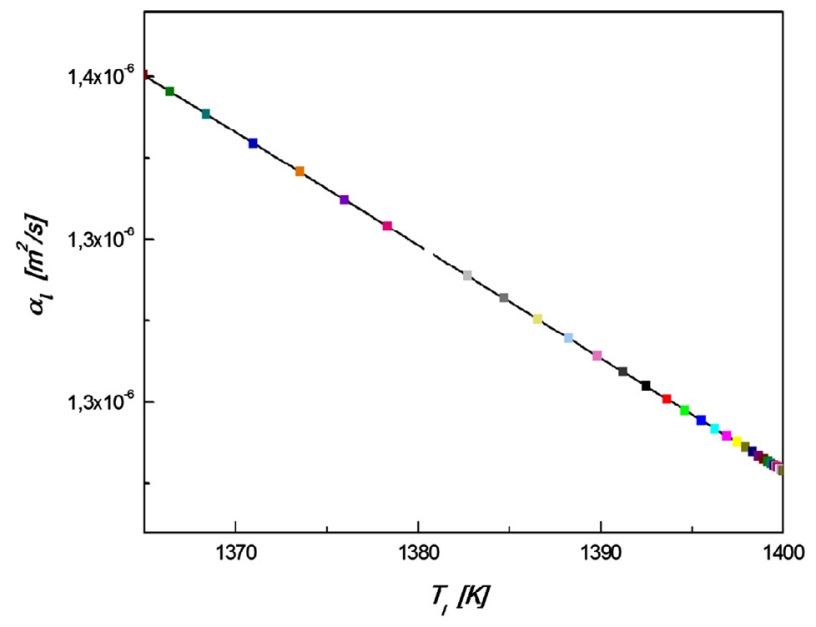

(b)

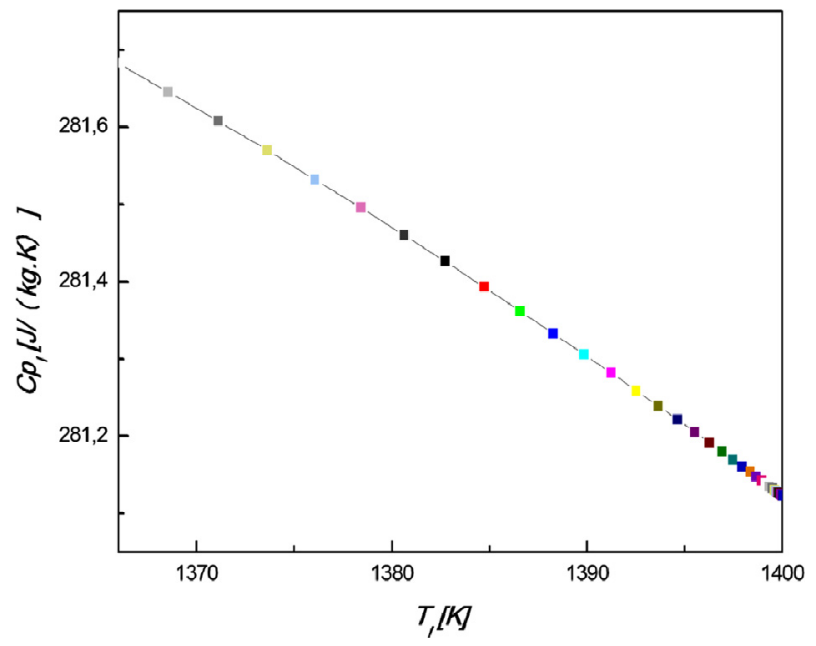

(d)

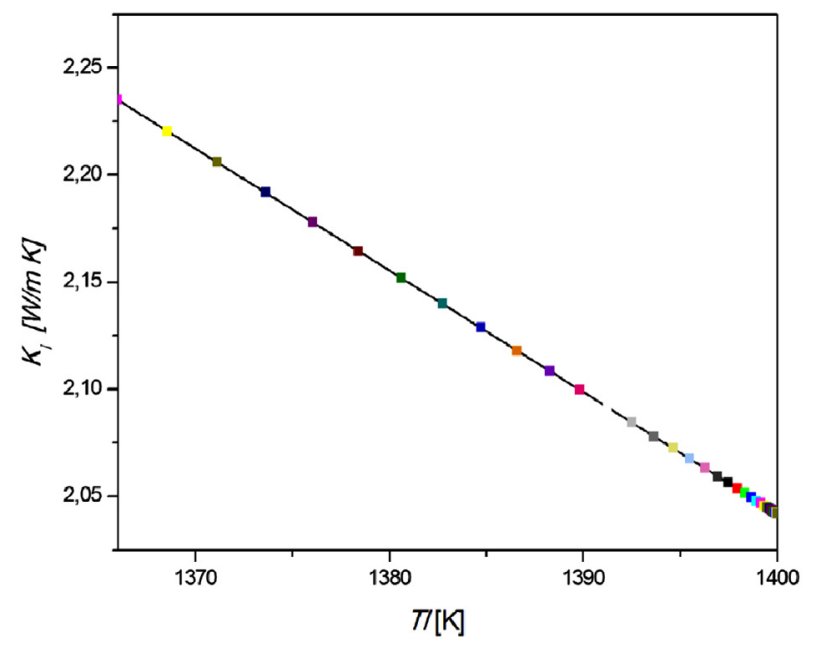

(f)

Figure 3. Thermal diffusivity, Specific heat and Thermal conductivity of CdZnTe relatively to the solid domain (a) and the liquid one (b), respectively for fixed parameters $T_{\mathrm{c}}=1200 \mathrm{~K}, T_{\mathrm{m}}=1364 \mathrm{~K}$, and $T_{\mathrm{h}}=1400 \mathrm{~K}$. 


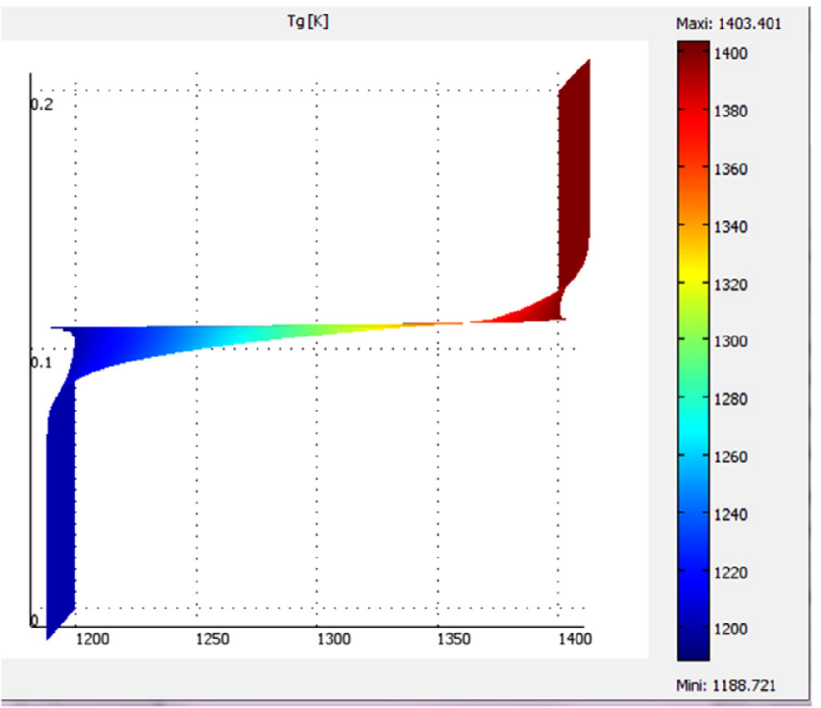

(a) Thermal profile

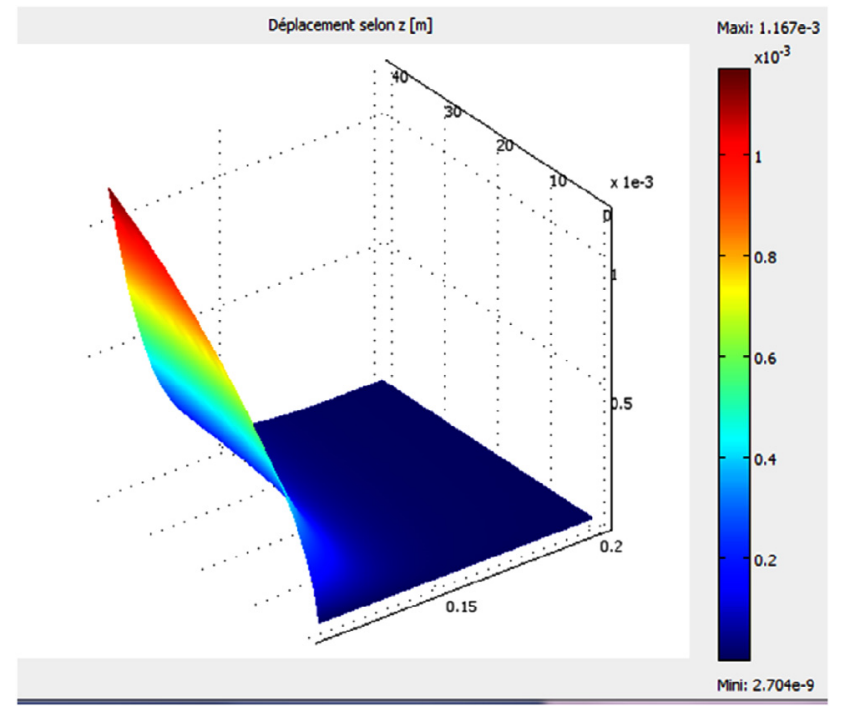

(b) Interface profile

Figure 4. Vizualisation of temperature field, and the spatial and temporal variations of the interface evolution in both solid and liquid domains.

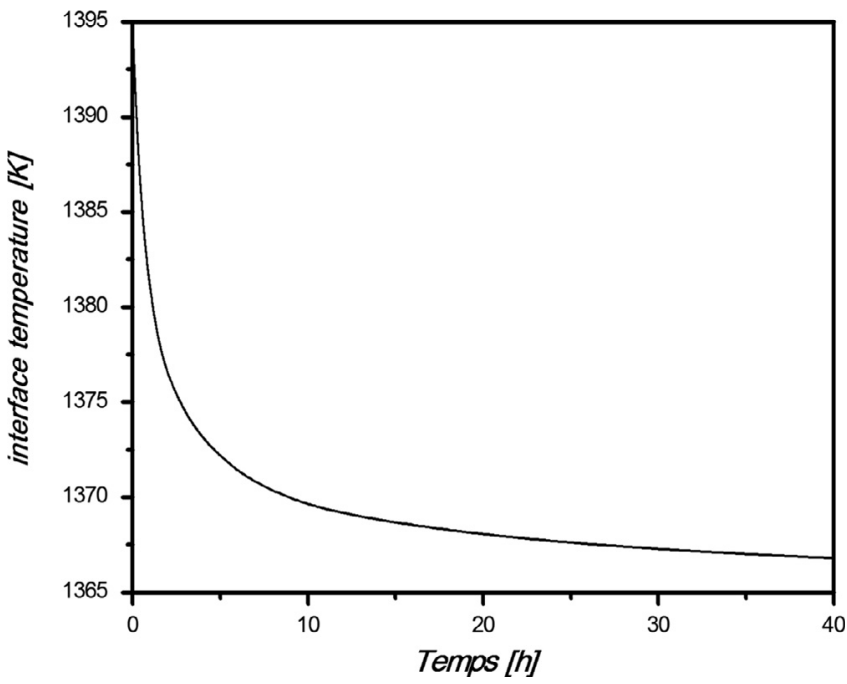

Figure 5. Temporal variation relative to the temperature at the interface for $T_{\mathrm{h}}=1400 \mathrm{~K}, T_{\mathrm{c}}=1200 \mathrm{~K}$, and $T_{\mathrm{m}}=1364 \mathrm{~K}$.

and thermal conductivity have not been well investigated. In fact, these thermophysical properties have important factors in the Stefan equation and therefore have an effect on the solidification, interface of $\mathrm{CdZnTe}$. It is worth mentioning that both governing parameters the temperature of the solid domain is not well studied in literature and the growth rate phenomena still need better understanding and we propose to address such issue in the present work.

From this point of view, the main idea of the current investigation is to control the shape interface, in solidification process. In particular, we will focus on the various parameters that affect the concavity and convexity of the interface that are also an important factor in crystal growth, namely, the thermal conductivity, temperature of the solid domain, and growth rate.

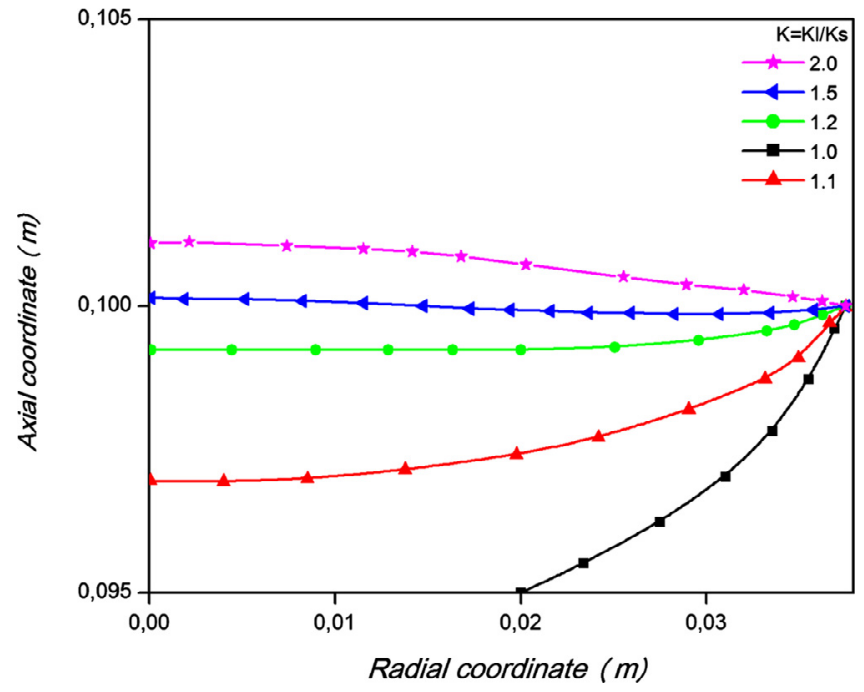

Figure 6. Influence of thermal conductivity ratio $\mathrm{K}=K_{\mathrm{l}} / K_{\mathrm{s}}$ on the interface shape for $T_{\mathrm{c}}=1200 \mathrm{~K}, T_{\mathrm{h}}=1400 \mathrm{~K}$, and $T_{\mathrm{m}}=1364 \mathrm{~K}$.

\section{Materials and methods}

\subsection{Physical properties of the materials}

The physical properties of CdZnTe are listed in Table 1 $[18,19]$. It should be noted that these properties depend on the given temperature, and they are in accordance with the thermal boundary conditions, for each domains.

\subsection{Physics model}

The Bridgman crystal system is schematically displayed in Figure 1a. The furnace uses a tapered heating element to establish a linear temperature profile Figure $1 \mathrm{~b}$. The ampoule is 


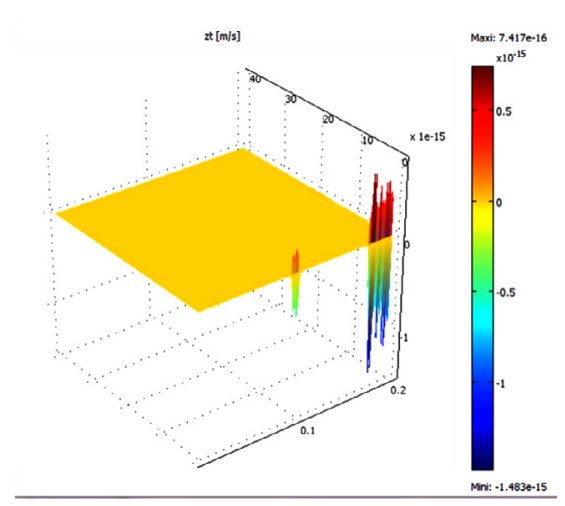

(a) $V_{\text {int }}=0$

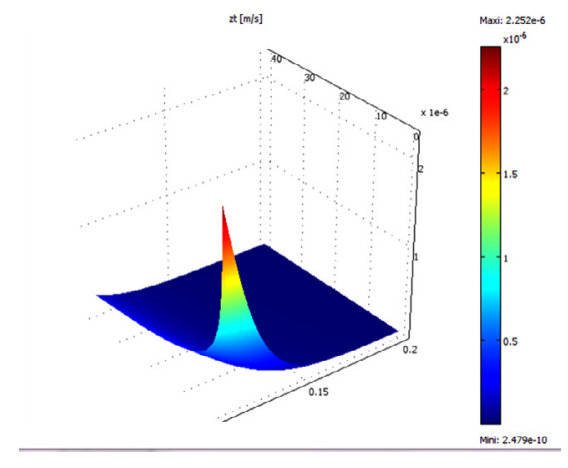

(d) $V_{\text {int }}=10^{-7}$

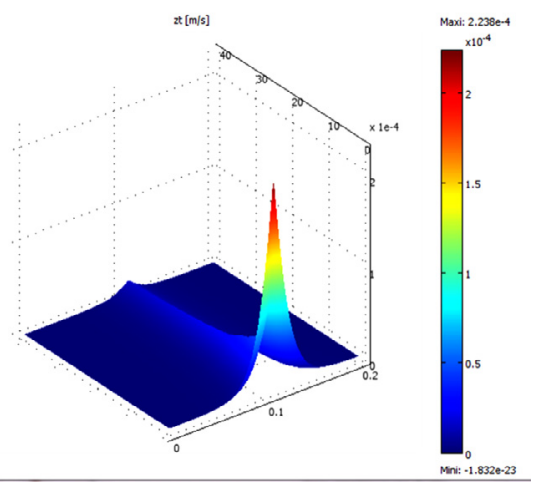

(b) $\mathrm{Vi}_{\text {nter }}=$ stefan equation

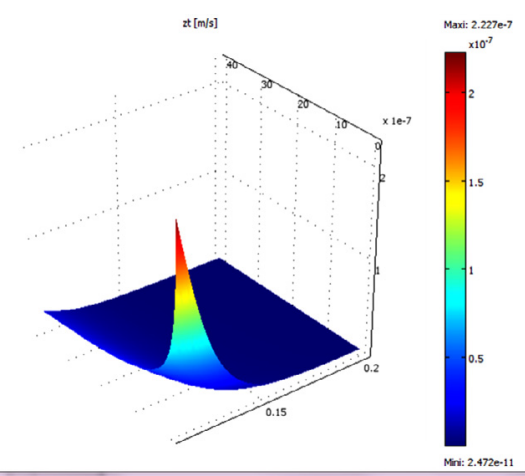

(e) $V_{\text {int }}=10^{-8}$

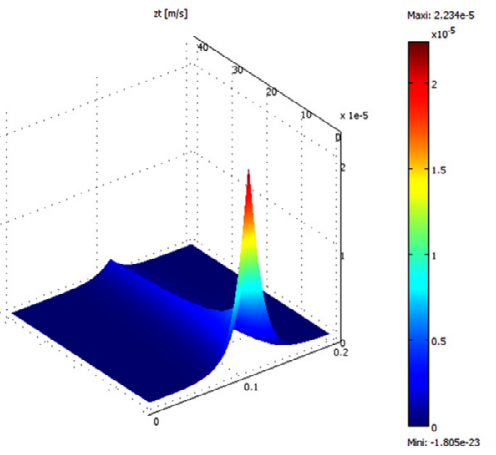

(c) $V_{\text {int }}=10^{-6}$

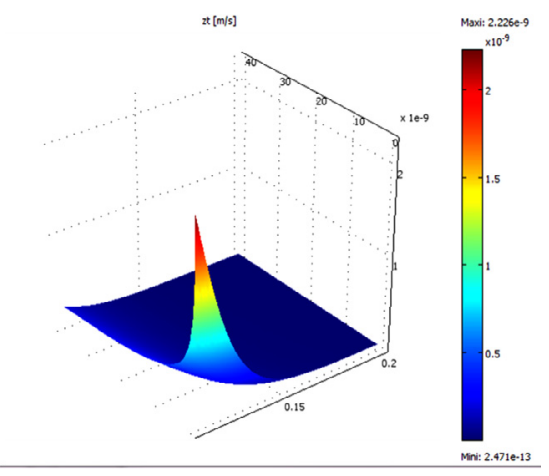

(f) $V_{\text {int }}=10^{-10}$

Figure 7. Temporal and spatial variations of isovalues relative to the interface velocity.

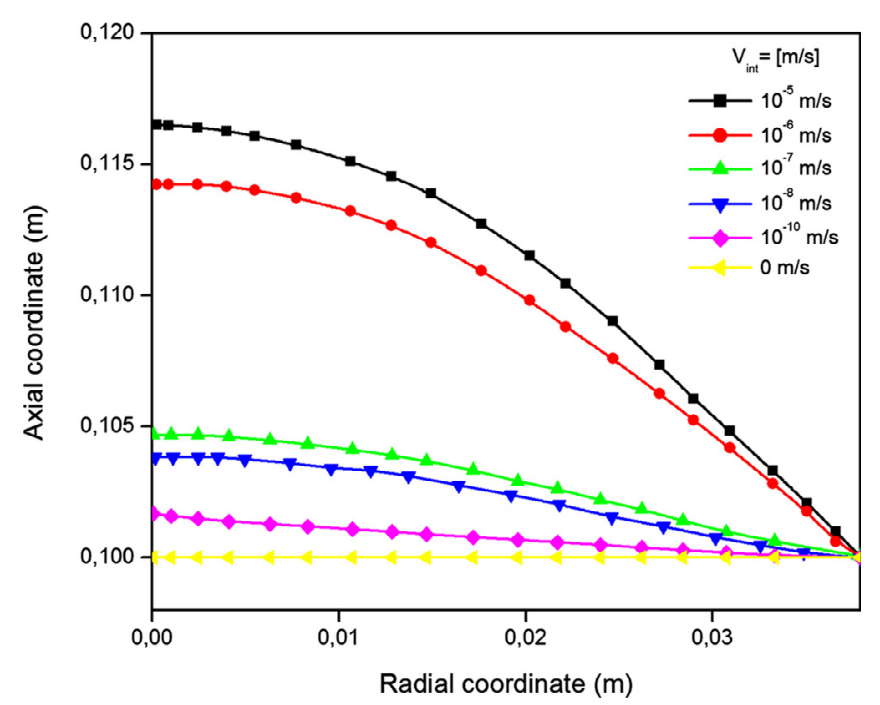

Figure 8. Effect of the interface velocity on the interface shape for $T_{\mathrm{c}}=1200 \mathrm{~K}, T_{\mathrm{h}}=1400 \mathrm{~K}$, and $T_{\mathrm{m}}=1364 \mathrm{~K}$.

axially positioned at the center of the growth furnace. During the process, the cylindrical ampoule containing the material in the solid and liquid phase is fixed at the constant rate $V_{t}=0$. Because of the symmetry of the system, the right half of Figure 1c is taken as the calculation domain only supposed.

There is only heat conduction between the melt and crystal.
We can assume that this problem can be considered as a two dimensional axis-symmetry one, the temperature field, the Stefan equations are expressed in the cylindrical coordinates system.

The energy equations for the melt and the crystal given by:

$$
\begin{aligned}
& \nabla\left(K_{1} \nabla T_{1}\right)-\rho C p_{1} \frac{\partial T_{1}}{\partial t}=0, \\
& \nabla\left(K_{\mathrm{s}} \nabla T_{\mathrm{s}}\right)-\rho C p_{\mathrm{s}} \frac{\partial T_{\mathrm{s}}}{\partial t}=0 .
\end{aligned}
$$

The shape of the solid-liquid interface is a compulsory controlling factor that allows producing a single crystal in vertical Bridgman enclosure. This interface shape is predicted by heat transfer and primarily by the direction of the heat flux near the interface.

This interface is governed by the Stefan's equation expressed as:

$$
\left(K_{\mathrm{s}} \nabla T_{\mathrm{s}}\right) \times n-\left(K_{1} \nabla T_{1}\right) \times n=\rho \Delta H V_{\mathrm{int}} .
$$

The aforementioned parameters in the above equations $K_{\mathrm{s}}$, $K_{\mathrm{l}}, \rho, C p_{\mathrm{l}}$, and $C p_{\mathrm{s}}$ are the thermal conductivities, the density, specifics heats, in solid (denoted by s) and liquid (denoted by 1), respectively. Here, $\Delta H$ expresses the latent heat and $V_{\text {int }}$ denoted the velocity at the interface. 


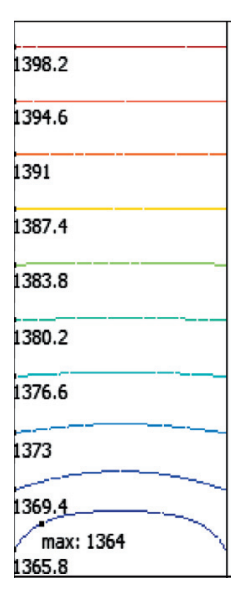

$\mathrm{Tc}=1200$

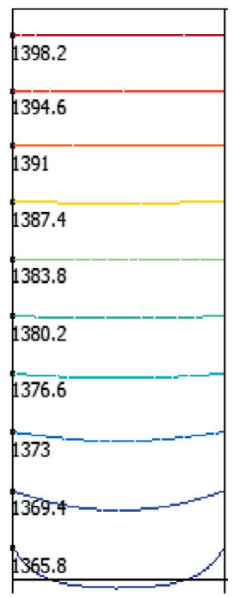

$\mathrm{Tc}=1284$

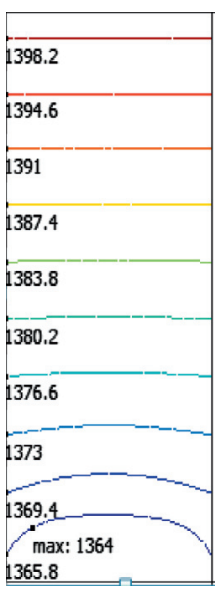

$\mathrm{Tc}=1224$

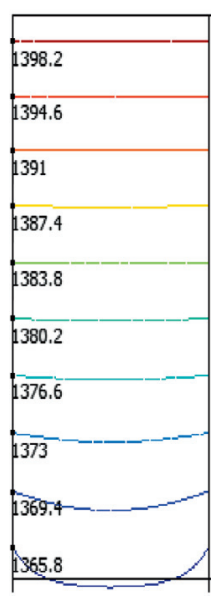

$\mathrm{Tc}=1324$

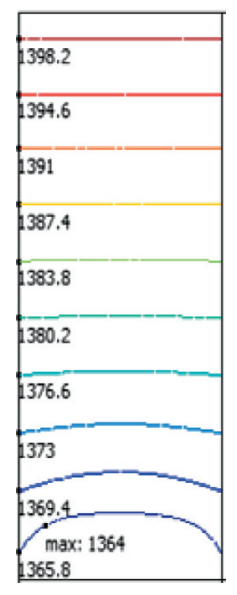

$\mathrm{T}=1244$

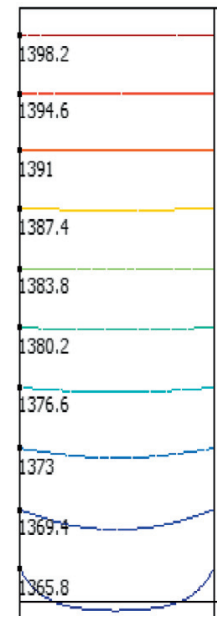

$\mathrm{Tc}=1344$

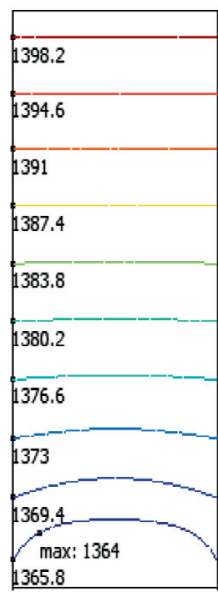

$\mathrm{T}=1264$

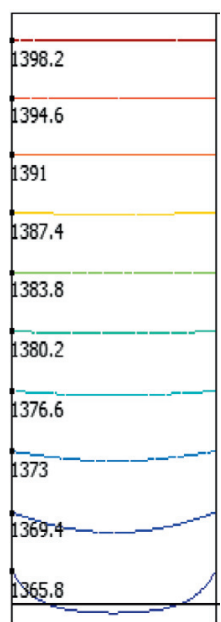

$\mathrm{Tc}=1354$

Figure 9. Isotherm patterns with respect to different values of the cold temperature $T_{\mathrm{c}}$ for $T_{\mathrm{h}}=1400 \mathrm{~K}$ and $T_{\mathrm{m}}=1364 \mathrm{~K}$.

The boundaries conditions corresponding to the temperature field are as follows: the left and right boundaries are kept adiabatic while the upper, the lower, and the interface position are maintained at $T_{\mathrm{h}}=1400 \mathrm{~K}, T_{\mathrm{c}}=1200$, and $T_{\mathrm{m}}=1364 \mathrm{~K}$, respectively.

\section{Numerical tools}

For solving the mathematical model industrial software has been used, namely COMSOL which essentially integrated MATLAB which includes mathematics and computation; data acquisition; modeling, algorithm development; simulation, and prototyping; data analysis, exploration, and visualization. On another hand, the MATLAB language is particularly well suited to designing predictive mathematical models and developing specific algorithms [20,21]. Where, these algorithms contain operations for linear algebra, partial differential, matrix manipulation, or differential equation solving, data analysis and statistics.

First results concerned the validation of the computational approach versus the mathematical model. For that a test validation has been defined and conducted. It refers to available experimental results with considering shaped solid/liquid interface.

\subsection{Test code validation}

The validation herein concerns the physical problem of CdZnTe solidification in Vertical Bridgman (BV). In fact, the choice of this configuration is based on the experimental data [22-24] for CdZnTe crystal growth: $R=0.0375 \mathrm{~m}$, $L=0.20 \mathrm{~m}$, the temperature of the Bridgman furnace the upper of them being at a higher temperature than the melting point while the lower one is below the melting point. Thus, the system is cooled from below, and the solidification process begins from the bottom of the ampoule.

We noticed the same shape interface between our numerical computations (Figure 2b) and experimental ones (Figure 2a) in which the authors [18] presented the half of longitudinal section of grown ingot of CdZnTe. In fact, they mentioned, that the interface shape at the end of growth is concave toward the crystal, with interface bending of $3 \mathrm{~mm}$ Figure 2a. The same results 


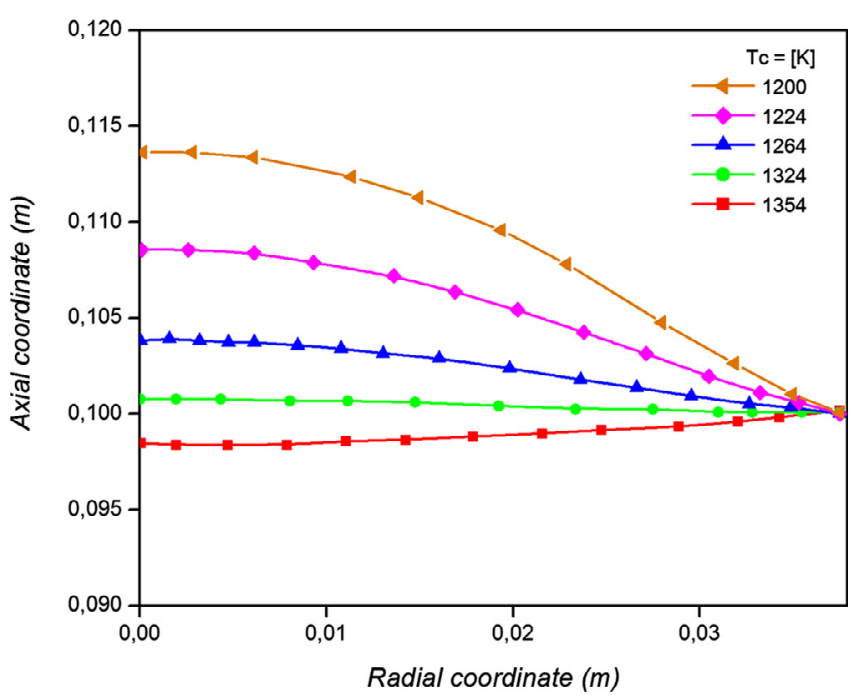

Figure 10. Effect of the cold temperature on the interface shape at fixed parameter values $T_{\mathrm{c}}=1200 \mathrm{~K}, T_{\mathrm{h}}=1400 \mathrm{~K}$, and $T_{\mathrm{m}}=1364 \mathrm{~K}$.

are shown numerically Figure $2 \mathrm{~b}$ and a good agreement is observed with those obtained by Sen et al. [18]. In fact, the same results are revealed by the current simulation in which the shape interface is concaved and the progress rate is found to be around $3 \mathrm{~mm}$ in $9 \mathrm{~h}$.

\section{Results and discussion}

Our results concerned the solidification process of CdZnTe in the Vertical Bridgman (VB) configuration. In fact, we will present the different parameters which affect the solidification of CdZnTe, with the aid of numerical simulations.

As aforementioned, one of the main ideas of our investigation is to present a general study of different parameters that affect the quality of the single CdZnTe crystal. Especially, we will give a great importance to study the thermo-physical properties of CdZnTe in each domain (solid or liquid) such as the thermal diffusivity $\alpha$, the specific heat $C p$ (at constant pressure), and the thermal conductivity, that all depend on the temperature and which are measured for both solid and liquid CdZnTe. In addition, thermal diffusivity is an important factor in the current investigation. In fact, not only it offers a convenient and accurate method of determining the thermal conductivity $(k)$, but also it is a crucial parameter in the conduction equation which governs heat transfer during crystal growth [18]. In addition, these parameters have a great impact on the Stefan equation that interferes in the solidification interface process.

In fact, as shown in Figures $3 \mathrm{a}$ and $3 \mathrm{~b}$ illustrating the profile of thermal diffusivity in both solid and liquid domains, we note that this diffusivity increases for temperature in the solid domain while it decreases with temperature in the liquid state. Indeed, one can note that the diffusivity goes through a sharp minimum in the region of melting point; the value in the liquid is higher than that in the solid just before the onset of melting. The same results are showed by Sen et al. [18], who treated the influence of thermal diffusivity for CdTe and Cd0.96Zn0.04Te.
However, their results are significantly different from those reported by Marina et al. [22] who claimed that thermal diffusivity increased sharply above the liquid temperature for $\mathrm{Hg}_{1-x} \mathrm{Cd}_{x} \mathrm{Te}$.

In the same context, we study the shape of the heat capacity shown in Figures $3 \mathrm{c}$ and $3 \mathrm{~d}$ in both the solid and liquid domain. Besides, as shown in Figure 3a, the heat capacity is observed to be progressively augmented in the solid domain until reaching a maximum of temperature, precisely at the value $T_{\mathrm{c}}=1260 \mathrm{~K}$. Consequently, by approaching the melting temperature, the profile of thermal capacity undergoes a decreasing trend until reaching a constant value in the liquid domain Figure $3 b$.

Similarly, we propose now to study the thermal conductivity and its influence on the interface shape and position as determined, in this case. In fact, we observed that the profile of thermal conductivity Figures $3 \mathrm{e}$ and $3 \mathrm{f}$ is similar to that relative to thermal diffusivity in both solid and liquid domain as revealed in Figures $3 \mathrm{a}$ and $3 \mathrm{~b}$. This makes good sense because thermal conductivity is proportional to thermal diffusivity which expression is defined by $\alpha=k / \rho C p$. In fact, it was found that the profile of solid thermal conductivity $K_{\mathrm{s}}$ is more enhanced compared to $K_{1}$ in liquid state.

Figure $4 \mathrm{a}$ shows the temperature according to the solidification of CdZnTe along the both liquid-solid state plotted as function of axial coordinate, where $z=-0.01 \mathrm{~m}$ denotes the bottom tip of solid domain, and $z=0.20 \mathrm{~m}$ corresponds to both liquid and liquid states. This thermal profile is based on two linear segments designed to yield a gradient in the melt and a steeper cooling rate in the crystal [25]. And, the visualization of interface evolution denoted by the Figure $4 \mathrm{~b}$, proved that is increased with increasing time.

Figure 5 shows the interface temperature parameter as function of time. In fact, as note that after the initial transient, the temperature presented a peak which decreases steadily until a mild increase near the end of the run. The decreasing peak temperature coincides with decreasing melt height and volume as growth occurs. It is worth mentioning that similar results have been revealed by Cerny et al. [26], which mentioned that the time development of interface temperatures reveals well the establishment of the pseudo-steady regime of crystal growth after $15 \mathrm{~h}$, and by Zang et al. [27] in the problem of solidification of CdZnTe crystal growth from the melt.

Thereafter, we will present the effect of the thermal conductivities ratio defined by $K$ in the shape interface. We found that it is of great importance, because it changes not only the sign of curvature and consequently the concavity and the convexity of the interface shape, but also the curvature magnitude. Therefore, it was argued that for $T_{\mathrm{h}}=1400 \mathrm{~K}, T_{\mathrm{c}}=1200 \mathrm{~K}$ and $T_{\mathrm{m}}=1364 \mathrm{~K}$, the parameter $K$ ensures the achievement of a convex interface when it is less than 1.1 and over which the interface becomes concave Figure 6. Therefore, it is to conclude that the conductivity ratio is of great importance in the CdZnTe solidification because it helps controlling the process of crystal growth.

Among the problems encountered in numerical simulation is the interface computation. These problems are generally called Stefan problems (Eq. (3)) that connects the axial temperature gradient in the liquid and the solid domain by the velocity growth. In fact, we devote this section to study the evolution of 
velocity interface for different values taken in the solidification of CdZnTe. The main issue in this section is to give a better visualization of the velocity growth and surface evolution which will allow to better understand the phenomenon of growth, especially the evolution and of the interface shape Figure 7 of great interest in this portion is to vary the velocity interface while keeping the speed of the bulb at zero, in other words the bulb is held fixed. The velocity profile depicted in Figure 7 demonstrates that the velocity value determined by the Stefan equation is strengthened compared to that computed during the growth of $\mathrm{CdZnTe}$ especially in the solidification of the material. This value is seen to be nearly $10^{-5} \mathrm{~m} / \mathrm{s}$ given by COMSOL after resolution and compilation of the Stefan equation while respecting the requirement for thermal boundary conditions $\mathrm{s}$ in both liquid and solid areas.

It was also noted that the decrease of the interface velocity from $10^{-5}$ to $10^{-10} \mathrm{~m} / \mathrm{s}$ has a great effect on the evolution and the interface shape which becomes progressively weakened in Figure 8 . Therefore one can conclude that the velocity variation is an important factor in the industrialization of crystal growth.

In this part we attempt to focus on predicting the effect of the variation of cold temperature $T_{\mathrm{c}}$ on the isotherms in the liquid state which they are affected by the advancement of the shape interface and consequently the concavity and the convexity magnitude. In fact, as we mentioned before in the introduction, although this factor is also important in the understanding of crystal growth industrialization, it has not been well investigated until now. As revealed from Figure 9, we can mention that the variation of $T_{\mathrm{c}}$ affects the concavity and the convexity of the interface. In fact, for $T_{\mathrm{c}} \in[1200 \mathrm{~K}-1264 \mathrm{~K}]$, the interface remains concave while it becomes convex for $T_{\mathrm{c}} \in[1265-1354 \mathrm{~K}]$ especially, when the cold temperature reaches the value $T_{\mathrm{c}}=1265 \mathrm{k}$ according to the fixed values $T_{\mathrm{h}}=1400 \mathrm{~K}, T_{\mathrm{m}}=1364 \mathrm{~K}$, and $R=0.037$. However, by increasing $T_{\mathrm{c}}$ over the value $1354 \mathrm{~K}$, the problem is seen to diverge because the temperature approaches the specific value of $T_{\mathrm{m}}$.

We can finally conclude that the variation of $T_{\mathrm{c}}$ is of great importance such as the thermal conductivities ratios and the velocity interface variation, and this parameter can help to solve some problems encountered during the growth of CdZnTe, especially the problem of the concavity and convexity of the interface shape which affects the quality of crystal growth.

Do not forget also that the purpose of any study is to solve the problems not only in the semiconductor factory, but also to obtain crystals of good qualities. Investigations must help to choose the value of the cold temperature which allow operators to obtain a concave or convex interface

In the same as mentioned in Figure 10 the variation of axial coordinate with radial coordinate for different values of cold temperature which reveals the same interpretation proved in Figure 9.

Our numerical findings may contribute in controlling the parameters for the growth process, and it represents a strong support to resolve difficulties for experimentalists.

\section{Conclusion}

In the current investigation, solidification of the singular CdZnTe in axisymetric Vertical Bridgman (VB) cavity is studied. All numerical computations are obtained using COMSOL Multiphysics software, which is based on finite elements method (FEM) with ALE (Arbitrary Lagrangian-Euleurian) formulation allowing treating the interface displacement. The visualization of temperature fields in terms of isotherms and spatial and temporal profiles and according to the solidification of CdZnTe is presented and discussed with the help of COMSOL to provide a better understanding of phase change. Especial attention has been attributed to study the thermo-physical properties of $\mathrm{CdZnTe}$ in each domain (solid or liquid) such as the thermal diffusivity $\alpha$, the specific heat $C p$ (at constant pressure), and the thermal conductivity, which are measured for both solid and liquid CdZnTe. In this context, it is found that iso-surfaces of thermal diffusivity in both solid and liquid domains show that diffusivity increases for temperature in the solid domain while it is lowered with temperature in the liquid state. Furthermore, the diffusivity goes through a sharp minimum in the region of melting point and the value in the liquid is higher than that in the solid just before the onset of melting. In addition, the heat capacity is observed to progressively augment in the solid domain until reaching a maximum of temperature, at exactly the value $T_{\mathrm{c}}=1260 \mathrm{~K}$. On another hand, it is found that the variation of $T_{\mathrm{c}}$ affects the concavity and the convexity of the interface. In fact, it is seen that for specific interval of $T_{\mathrm{c}}$ the interface remains concave while it becomes convex for another one. Concerning the effect of the interface velocity variation, it is found that the decrease of the interface velocity from $10^{-5}$ to $10^{-10} \mathrm{~m} / \mathrm{s}$ has a great influence on the evolution and the interface shape which becomes progressively weakened. In this vein, analysis of the results shows that the velocity variation is seen to be an important factor in the industrialization of crystal growth.

Acknowledgements. The fist author thanks Profs. R. Bennacer (ENS Cachan) and A. Aydi (university of sfax) for fruitful discussion.

\section{References}

1. Chang CE, Wilcox WR. 1974. Control of interface shape in the vertical Bridgman-Stockbarger technique. Journal of Crystal Growth, 21, 135-140.

2. Sen S, Wilcox WR. 1975. Influence of crucible on interface shape, position and sensitivity in the vertical BridgmanStockbarger technique. Journal of Crystal Growth, 28, 26-40.

3. Fu T-W, Wilcox WR. 1980. Influence of insulation on stability of interface shape and position in the vertical BridgmanStockbarger technique. Journal of Crystal Growth, 48, 416-424.

4. Naumann RJ. 1982. An analytical approach to the thermal modeling of Bridgman-type crystal growth. II. Two-dimensional analysis. Journal of Crystal Growth, 58, 569-584.

5. Naumann RJ, Lehoczky SL. 1983. Effect of variable thermal conductivity on isotherms in Bridgman growth. Journal of Crystal Growth, 61, 707-710. 
6. Adornato P, Brown RA. 1987. Convection and segregation in directional solidification of dilute and non-dilute binary alloys: effects of ampoule and furnace design. Journal of Crystal Growth, 80, 155-190.

7. Wang CA, Witt AF, Carruthers JR. 1984. Analysis of crystal growth characteristics in a conventional vertical Bridgman configuration. Journal of Crystal Growth, 66, 299-308.

8. Jasinski T, Witt AF. 1985. On control of the crystal melt interface shape during growth in a vertical Bridgman configuration. Journal of Crystal Growth, 71, 295-304.

9. Jasinski T, Witt AF, U.S. Patent: Apparatus for Growing Crystals, 1986.

10. El Ganaoui M, Lamazouade A, Bontoux P. 2002. Computational solution for fluid flow under solid/liquid phase change conditions. Computers \& Fluids, 31, 539-556.

11. Crank IJ. 1984. Free and moving boundary problems. Clarendon Press: Oxford, UK.

12. Voller R, Prakash C. 1987. A fixed grid numerical modelling methodology for convection diffusion mushy region phase change problems. International Journal of Heat and Mass Transfer, 30(8), 1709-1719.

13. Bennon WD, Incropera FP. 1987. A continuum model for momentum, heat and species transport in binary solid-liquid phase change systems: 1. Model formulation. International Journal of Heat and Mass Transfer, 30(10), 2161-2170.

14. Voller R, Cross M. 1980. Accurate solutions of moving boundary problems using the enthalpy method. International Journal of Heat and Mass Transfer, 24, 545-556.

15. El Ganaoui M. Modélisation numérique de la convection thermique instationnaire en présence d'un front de solidification déformable. Thèse de l'Université d'Aix-Marseille, Octobre, 1997.

16. El Ganaoui M, Mazhorova OS, Bontoux P. 2000. Computer simulation of pure and alloys melt growth. Microgravity Quart Rev, 7(4), 171-178.
17. Elganaoui M, Bontoux P, Morvan D. 1999, Localisation d'un front de solidification en interaction avec un bain fondu instationnaire, Paris: CR Acad Sciences, Série II b, t. 327, p. $41-48$.

18. Sen S, Konkel WH, Tighe SJ, Bland LG, Sharma SR, Taylor RE. 1988. Crystal growth of large-area single-crystal CdTe and CdZnTe by the computer-controlled vertical modified-Bridgman process. Journal of Crystal Growth, 86, 111.

19. Bruder M, Figgemeier H, Schmitt R, Maier H. 1993. Mat. Res. Eng. B, 16, 40.

20. Kuppurao S, Brandon S, Derby JJ. 1995. Journal of Crystal Growth, 155, 93.

21. MATLAB. The Language of Technical Computing - Programming Version 7, MA, USA: The Mathworks, Inc, 2005

22. Marchenko MP, Golyshev VD, Bykova SV. 2007. Investigation of $\mathrm{Cd}_{1-\mathrm{x}} \mathrm{Zn}_{\mathrm{x}} \mathrm{Te}$ composition inhomogeneity at crystal growth by AHP-method. Journal of Crystal Growth, 303, 193-198.

23. Huang ZH, Conway PP, Thomson RC, Dinsdale AT, Robinson JAJ. 2008. A computational interface for thermodynamic calculations software MTDATA. Computer Coupling of Phase Diagrams and Thermochemistry, 32, 129-134.

24. Bruder M, Figgemeier H, Schmitt R, Maier H. 1993. Mat. Res. Eng. B, 16, 40.

25. Fang HS, Wang S, Zhou L, Zhou NG, Lin MH. 2012. Influence of furnace design on thermal stress during directional solidification of multicristalline silicon. Journal of Crystal Growth, 346, 5-11.

26. Cerny C, Kalbac A, Prikryl P. 2000. Computational modeling of $\mathrm{CdZnTe}$ crystal growth from the melt. Computational Materials Science, 1734-1760.

27. Zhang N, Yeckel A, Derby JJ. 2012. Maintaining convex interface shapes during electrodynamic gradient freeze growth of cadmium zinc telluride using a dynamic, bell-curve furnace profile. Journal of Crystal Growth, 355, 113-121.

Cite this article as: Jamai $\mathrm{H}$, Pateyron $\mathrm{B}$, Sammouda $\mathrm{H}$ \& El Ganaoui M: Numerical simulation of vertical Bridgman solidification of CdZnTe. Int. J. Simul. Multisci. Des. Optim., 2014, 5, A23. 